\title{
Darwin-GENIE: a nulling instrument at the VLTI
}

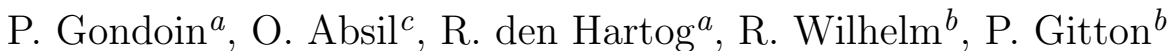

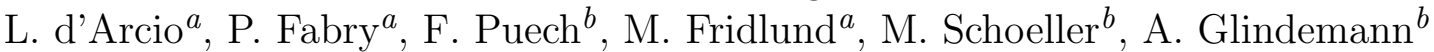 \\ E. Bakker ${ }^{d}$, A. Karlsson ${ }^{a}$, A. Peacock ${ }^{a}$, S. Volonte ${ }^{a}$, F. Paresce $^{b}$, A. Richichi ${ }^{b}$ \\ ${ }^{a}$ European Space Agency, P.O. Box 299, 2200AG Noordwijk, The Netherlands \\ ${ }^{b}$ European Southern Observatory, D-85748 Garching bei Munchen, Germany \\ ${ }^{c}$ University of Liege, Faculte des Sciences, Liege, Belgium \\ ${ }^{d}$ Leiden observatory, University of Leiden, The Netherlands
}

\begin{abstract}
Darwin is one of the most challenging space projects ever considered by the European Space Agency (ESA). Its principal objectives are to detect Earth-like planets around nearby stars and to characterise their atmospheres. Darwin is conceived as a space "nulling interferometer" which makes use of on-axis destructive interferences to extinguish the stellar light while keeping the off-axis signal of the orbiting planet. Within the frame of the Darwin program, the European Space Agency (ESA) and the European Southern Observatory (ESO) intend to build a ground-based technology demonstrator called GENIE (Ground based European Nulling Interferometry Experiment). Such a ground-based demonstrator built around the Very Large Telescope Interferometer (VLTI) in Paranal will test some of the key technologies required for the Darwin Infrared Space Interferometer. It will demonstrate that nulling interferometry can be achieved in a broad mid-IR band as a precursor to the next phase of the Darwin program. The instrument will operate in the L' band around $3.8 \mu \mathrm{m}$, where the thermal emission from the telescopes and the atmosphere is reduced. GENIE will be able to operate in two different configurations, i.e. either as a single Bracewell nulling interferometer or as a double-Bracewell nulling interferometer with an internal modulation scheme.
\end{abstract}

\section{INTRODUCTION}

Understanding the principles and processes that created the Earth, and allowed the development and evolution of life forms to take place is a key scientific objective which has been brought as a very high priority to the attention not only of the European Science Community but also of the European Space Agency. In particular, the successful detection of Earth-like planets possessing environments benign to life would answer central questions such as "How unique is the Earth as a planet?" and "How unique is life in the Universe ?". To achieve these objectives, the Darwin mission of the European Space Agency (Fridlund 2000; Fridlund \& Gondoin 2002) will survey a large sample of nearby stars and search for Earth-size planets within their "habitable zone". Darwin will measure their spectra in order to infer the presence of an atmosphere and search for biomarkers.

Detection of Earth-size bodies orbiting nearby stars is extremely difficult because of the weak planetary signal emitted within a fraction of an arcsecond from an overwhelmingly bright star. A solar type star outshines an Earth size planet by a factor of more than $10^{9}$ in the visible wavelength range (see Fig.2). In the infrared spectral range, where the planet's thermal emission increases and the star's emission decreases, the contrast is still higher than $10^{6}$. Only the planetary signal, a millionth of the stellar light, should remain in the input feed of a spectrograph in order to register a planet spectrum in a reasonable time. To accomplish such an extinguishing of light at the relevant spatial scales, the technique of "nulling interferometry" has been selected for Darwin. By applying suitable phase shifts between different telescopes in an interferometric array, destructive interference can be achieved on the optical axis of the system in the combined beam while interference is constructive for small off-axis angles. The principle of nulling interferometry for a simple two telescope Bracewell interferometer (Bracewell 1978) is described in Fig. 1. The equivalent transmission map of the nulling interferometer is a set of interference peaks with a sharp null in the centre. By placing the central star under this null, and adjusting the interferometer baseline to the required angular resolution, planets can be detected in the "habitable zone". The 

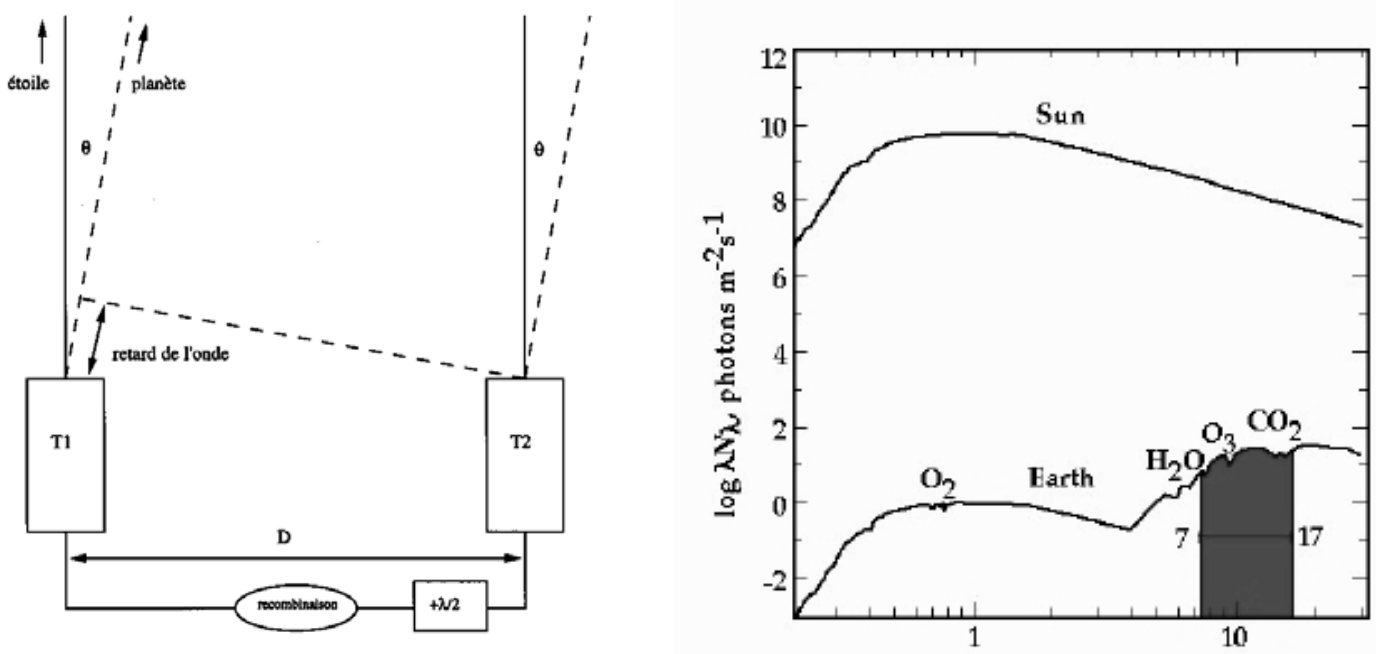

Figure 1. Left: principle of a two telescopes Bracewell interferometer. Right: contrast between the solar flux and the Earth as a function of wavelength in microns. Adapted from Angel et al. (1986).

actual shape and transmission properties of the pattern around the central null depend on the configuration and the distance between the telescopes.

A key issue for Darwin is the nulling interferometry technique. Laboratory breadboards with star-planet simulators have evaluated the performance of the nulling interferometric technique (Barillot 2003; Flatscher 2003) in narrow telecommunication band around $1.65 \mu \mathrm{m}$ where off-the-shelf components are available. The follow-up activity is to adapt their design to the mid-IR band and test the nulling interferometry technique on astronomical targets in Darwin representative operating conditions. The Very Large Telescope Interferometer (VLTI; Schöller \& Glindemann 2003) at the European Southern Observatory (ESO) is the most appropriate infrastructure on-ground to perform such a test. Hence, ESA and ESO have initiated a definition study for a Groundbased European Nulling Interferometry Experiment which will operate in the central laboratory of the VLTI at Mount Paranal (Chile). This experiment is called Darwin-GENIE. The definition study is assessing the technical feasibility of the experiment with the aim to establish its design, performance, programmatics and cost. If successful, the definition study will be the base for ESA and ESO to move into the hardware development, integration and exploitation phases of the GENIE project.

\section{GENIE OBJECTIVES}

\subsection{Demonstration of Darwin technology}

The primary objective of the Darwin-GENIE nulling experiment (Gondoin et al. 2003) is to gain experience on the design, manufacture and operation of a nulling interferometer using Darwin representative concept and technology. Nulling tests with the highest rejection factor on single stars or close binaries in broad mid-IR spectral bands will achieve this objective with the limitations imposed by the turbulence and infrared background of the Earth atmosphere. The Darwin-GENIE experiment will combine all optical functions foreseen into the future Darwin Infrared Space Interferometer. It will benefit from the existing VLTI infrastructure, including the telescope adaptive optics, the delay lines, the fringe sensors and the beam combiner laboratory. The overall performance of the instrument will heavily depend on the performance of all VLTI subsystems and in particular on the adaptive optics and co-phasing subsystems. The GENIE optical bench within the VLTI laboratory will provide the functions specific to the nulling interferometry technique, namely photometry and amplitude control, polarization matching, phase shifting, beam combination and internal modulation, spatial filtering, spectrometry, detection, electronics and cryogenics. The architecture of Darwin-GENIE is currently 

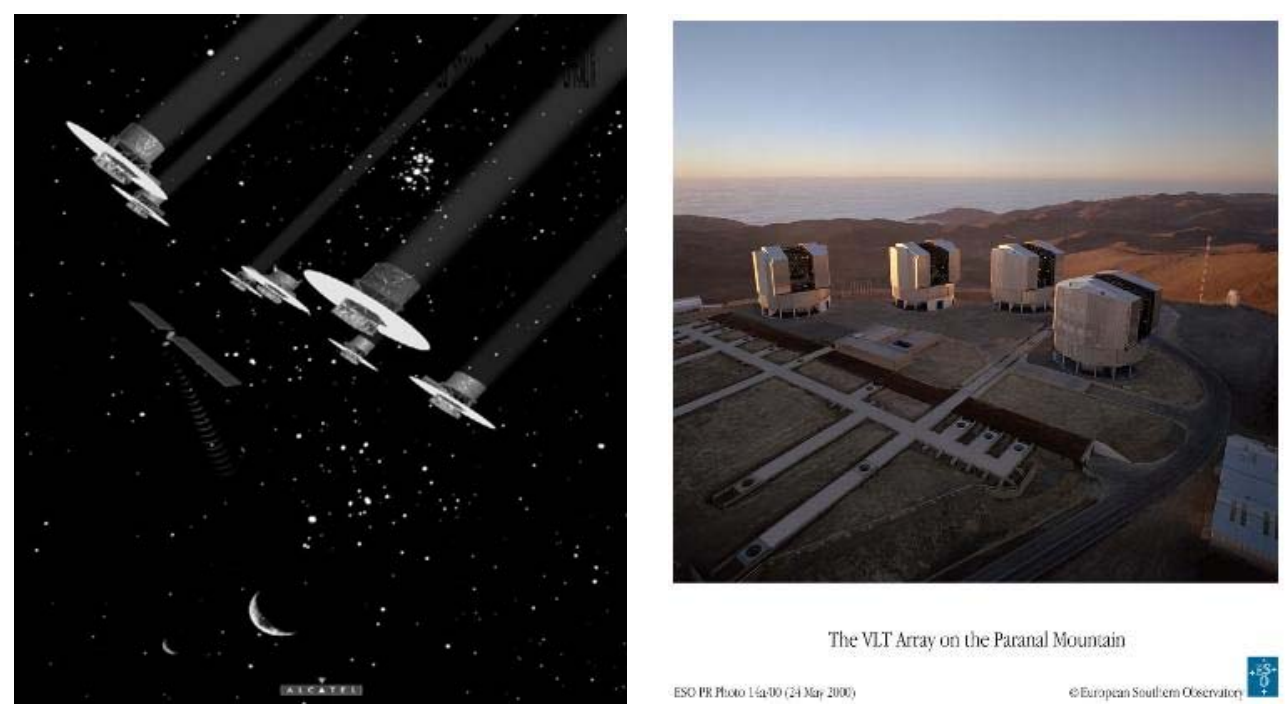

Figure 2. Left: Artist view of the Darwin Space Interferometer orbiting at Sun-Earth L2 point (courtesy Alcatel Space Division). Right: the ESO Very Large Telescope Interferometer with its four 8.2 meter telescopes and its central interferometric laboratory.

beeing defined taking into account the ESO VLTI interface characteristics and the output of the ESA Darwin tewchnology research program.

\subsection{Preparation of the Darwin science program}

Darwin science program aims to detect Earth-like planets around nearby stars, to determine their characteristics, to analyse the composition of their atmospheres and to assess their ability to sustain life as we know it. An important information for a proper design of the direct planet detection experiment of Darwin is the frequency of Earth-like planets around G, K and M dwarfs. The COROT space mission will detect massive bodies around cool stars by occultation and will thus provide this information. This mission is thus to be considered as a true precursor to Darwin. A second objective of GENIE is also to prepare the Darwin science program through a systematic survey of Darwin candidate targets (Eiroa et al. 2003). The solar zodiacal cloud, a sparse disk of 10-100 $\mu$ diameter silicate grains, is the most luminous component of the solar system after the Sun. Its optical depth is only $\approx 10^{-7}$, but a patch of the solar zodiacal cloud $0.3 \mathrm{AU}$ across has roughly the same emitting area as an Earth sized planet. Similar and even brighter clouds may be common in other planetary systems and present a severe obstacle for the direct detection of extra-solar terrestrial planets. A systematic survey of Darwin candidate targets will screen-out those stars for which circumstellar dust prevents the detection of Earth-like planets. Bright exozodiacal clouds are easier to detect than extra-solar terrestrial planets, but finding an exozodiacal cloud is still difficult. The total emission from our zodiacal cloud is no more than $10^{-4}$ of the Sun's at any wavelength. Photometric surveys like the Infrared Astronomical Satellite (IRAS) survey can only detect exozodiacal clouds that are > 500 times as optically thick as the solar clouds (Backman \& Parece 1993). Attempts to spatially resolve faint exozodiacal clouds with single-dish telescopes in the mid-infrared and near infrared (Küchner \& Brown 2000) have not yielded better detection limits. A Bracewell interferometer using two ESO VLTI 8m Unit Telescopes (UT) could provide better performance.

\section{GENIE CONFIGURATIONS}

GENIE will be able to operate in two different configuration, ie. either as a single Bracewell nulling interferometer or as a double-Bracewell nulling interferometer with an internal modulation scheme. The single Bracewell interferometer will be realized by combining the light from two Very Large Telescopes (UTs) or from two 1.8 


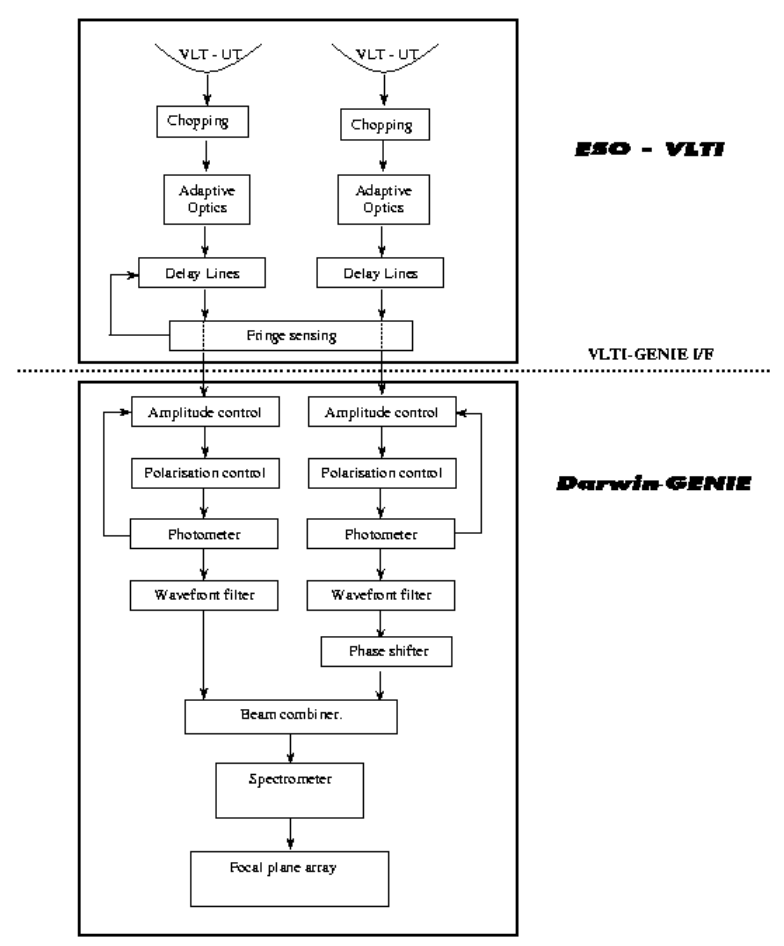

Figure 3. Functional description of GENIE.

m Auxiliary Telescopes (ATs) with a phase shift of $\pi$. The transmission map T( $\theta, \phi)$ (see Fig.4) of a diffraction limited Bracewell interferometer with two circular entrance apertures can be expressed as a function of wavelength, telescope diameter $\mathrm{D}$, and projected baseline B as follows:

$$
T(\theta, \phi)=2 \times \frac{J_{1}(\pi \theta D / \lambda)}{\pi \theta D / \lambda^{2}} \times \sin ^{2}(\pi \theta \cos (\phi) B / \lambda)
$$

$\theta, \phi$ are respectively the angular distance to the boresight and the azimut angle in the plan of the sky with respect to the projected baseline. Eq.(1) assumes a diffraction limited operation without any residual wavefront error, with a perfect $\pi$ phase shift between the two arms of the interferometer and equal amplitudes of the recombining fields. In practice, the central null of the transmission map will be degraded by amplitude and optical path differences (OPD) between the two arms of the interferometer that are not perfectly corrected.

Also, wavefront distorsions induced by atmospheric turbulence will only be partially compensated by the adaptive optics. Spatial filtering of the high frequencies wavefronts errors by monomode optical fibres or waveguides will be required (Ollivier \& Mariotti 1997). The coupling efficiency in the fibre will be variable (Shaklan \& Roddier 1988) depending on the fluctuating wavefront and pointing errors. Hence, the envelope of the transmission map of the interferometer will significantly depart from a Bessel function and will be highly time variable. In the Gaussian approximation, the optimum injection efficiency into a fibre is found for an aperture ratio such that the diffraction limited image has approximately the size of the core (Ruilier 1998). The 


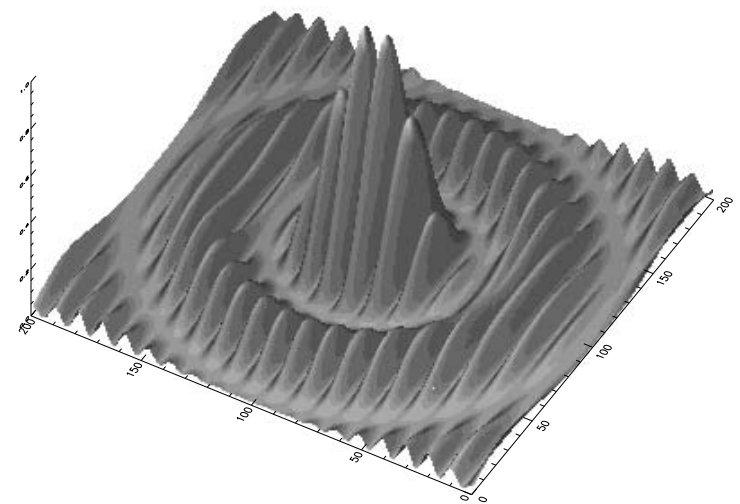

Figure 4. Transmission map of a diffraction limited Bracewell interferometer with circular entrance apertures.

geometrical extent of the beam seen by the fibre is then $S \Omega \approx \lambda^{2}$. The IR thermal background contribution is then proportional to $S \Omega \approx \lambda^{2}$ while the signal included into the field-of-view of the interferometer is proportional to the collecting area. Hence, the ratio signal to noise of an exozodiacal dust cloud observed in the mid-IR by a Bracewell interferometer is proportional to its collecting area and the largest telescopes shall be used.

The output signal of a Bracewell interferometer pointing to a star surrounded by a dust cloud can be expressed as follows:

$$
S(\theta, \phi)=A_{\mathrm{eff}} \int_{\theta} \int_{\phi} T(\theta, \phi) O(\theta, \phi) d \theta d \phi+B g d
$$

where $T(\theta, \phi)$ is the transmission map of the interferometer degraded by residual amplitude fluctuations and optical path differences between the two interferometer arms. $O(\theta, \phi)=O_{\mathrm{s}}(\theta, \phi)+O_{\mathrm{z}}(\theta, \phi)$ is the sum of the brightness of the star and of the exozodiacal dust disk. Bgd is the incoherent background signal which includes the thermal emission from the sky and from the telescope and instrument optics.

For background subtraction, GENIE will also use the internal chopping method similar to the one proposed in the context of the Keck nuller (Serabyn 2003). In this configuration, the image pupils of each of two UT telescopes will be divided in two parts in the VLTI beam combination laboratory. Each half-pupil of one telescope will be recombined destructively with the corresponding half-pupil of the other telescope, such that two Bracewell interferometers with parallel baselines are formed (see Fig.5). Their nulled output will be then combined with a $\pm \pi / 2$ phase shift. Chopping will thus be carried out at high frequencies by alternately registering the signal from these two outputs (see Fig. 6).

\section{THE TECHNICAL CHALLENGES}

\subsection{The stellar leakage}

The transmission map of a Bracewell interferometer exhibits a narrow null with a $\theta^{2}$ transmission on axis. Eq. 2 indicates that the high spatial resolution of a two telescopes interferometer with a $\approx 50 \mathrm{~m}$ baseline does not completely reject the starlight on axis specially when stars are closer than 50 pc. A residual stellar signal 


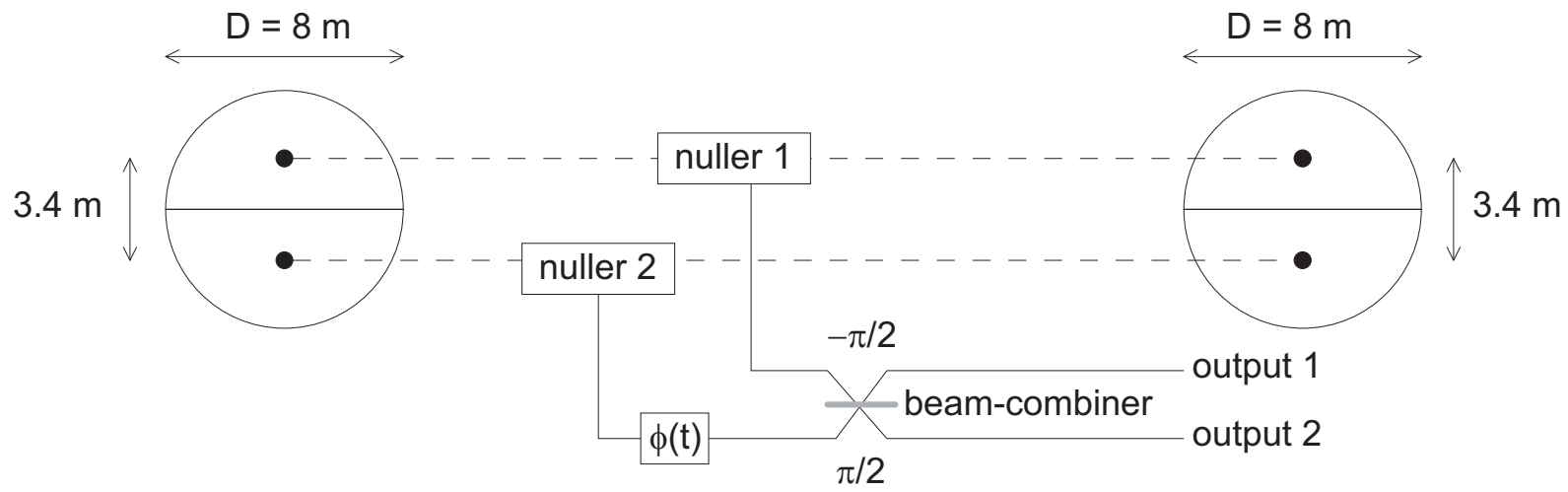

Figure 5. Principle of a double Bracewell configuration using two telescopes with split pupils.

$S_{\text {leak }}$ leaks through the transmission map. It can be expressed as a function of the operating wavelength $\lambda$, interferometer baseline $B$ and angular radius $\theta_{\mathrm{s}}$ of the star as follows:

$$
S_{\text {leak }}=A_{\text {eff }} \int_{\theta} \int_{\phi} T(\theta, \phi) O_{\mathrm{s}}(\theta, \phi) d \theta d \phi
$$

i.e. for a diffraction limited Bracewell interferometer:

$$
S_{\text {leak }}=A_{\text {eff }} \times O_{\mathrm{s}} \times\left(\pi^{2} / 8\right) \times\left(\theta_{\mathrm{s}} B / \lambda\right)^{2}
$$

In practice the transmission map is distorted and fluctuating rapidling as a function of time. Unequal amplitudes between the arms of the interferometer affect the depth of the null. Variations of the optical path difference (OPD) between the two telescopes induce random displacements of the central dark fringe. Hence the null signal is fluctuating on time scale that are short compared with typical integration times. When averaged over the detector frame read-out time $\left(f_{\text {ro }}^{-1}\right)$, one major effect of the stellar leakage is to raise the depth of the null to an average stellar leakage $<S_{\text {leak }}>$ that can be estimated as follows:

$$
\frac{<S_{\text {leak }}>}{F_{\mathrm{s}} \times f_{\mathrm{ro}}^{-1}}=\frac{\alpha^{2}}{4}+\frac{4 \alpha \Delta}{3 \pi}+\left(\frac{\pi \sigma_{\text {piston }}}{\lambda}\right)^{2}+\sigma_{a}^{2}+\frac{\Delta^{2}}{4}
$$

where $\alpha=\frac{\pi \theta_{\mathrm{s}}}{\lambda / B} \cdot \sigma_{a}$ is the rms fluctuation of the relative amplitude between the two arms of the interferometer after spatial filtering. $\sigma_{\text {piston }}$ is the rms fluctuation of the piston i.e the residual rms OPD fluctuation after correction by a delay line operated by a fringe sensor unit in a close loop system. $\Delta$ is a phase shift residual depending on wavelength which describe dispersion effects. In addition to an average offset of the null depth, a major effect of the stellar leakage is to induce important noise contributions. One contribution is the photon noise of the mean stellar leak $<S_{\text {leak }}>$ which adds onto the photon noise of the background and of the exozodiacal dust clouds. This degrades the ratio signal to noise of the exo-zodiacal dust disk measurements (see Eq. 7). A second source of noise is the erratic fluctuation $\sigma_{\text {leak }}$ of the stellar leakage due to residual variations at high frequencies of the amplitude and optical path difference between the two arms of the interferometer. The rms fluctuation of the stellar leakage are expressed as follows:

$$
\sigma_{\text {leak }}=F_{\mathrm{s}} \times \frac{8 \alpha}{3 \pi} \times \sqrt{\left(\frac{\pi \sigma_{\text {piston }}}{\lambda}\right)^{2}+\sigma_{a}^{2}} \times f_{\mathrm{ro}}^{-1}
$$

The effect of the stellar leakage fluctuations and photon noise on the ratio signal to noise of the dust disk flux measurements can therefore be expressed as follows: 


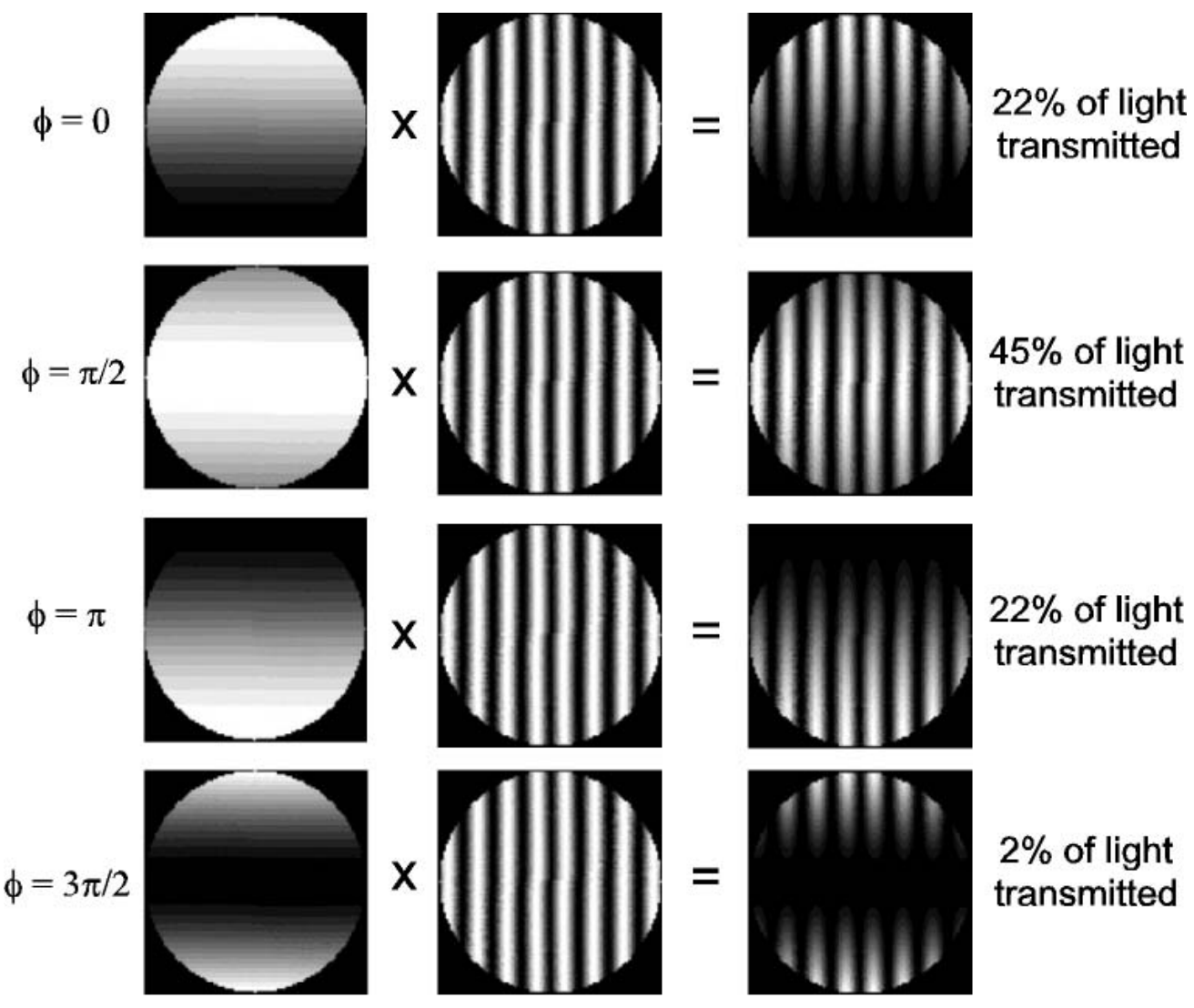

Figure 6. Transmission maps of a double Bracewell interferometer using two telescopes with split pupils. The transmission of the exo-zodiacal light around the target star is modulated by the variable phase shift between the two Bracewell interferometers while the incoherent background contribution is independent of the phase shift.

$$
S / N=\frac{S_{z} \sqrt{\Delta \lambda \Delta t}}{\sqrt{B g d+S_{z}+<S_{\text {leak }}>+\sigma_{\text {leak }}^{2}}}
$$

Photon noise and fluctuations of the stellar leakage are major limitations for the detection of exozodiacal dust clouds using a groundbased nulling interferometer. Eq.(4) shows that the stellar leakage of a Bracewell interferometer and therefore its contributions to the noise can be reduced by operating with short baselines. According to this criterion, the detection of exo-zodiacal dust clouds at VLTI would be most efficient with a Bracewell interferometer combining two large $8.2 \mathrm{~m}$ UT telescopes with the shortest VLTI baselines (e.g 46 or 56 meters).

\subsection{The thermal IR background}

In the $\mathrm{N}$ band, the infrared background flux in the interferometer field of view is about 300 times larger than the flux from a Sun-like star located at 10 parsecs. Fig.7 (left) shows the estimated nulling performance of the GENIE instrument with a $46 \mathrm{~m}$ baseline in the $\mathrm{N}$ band as a function of the star distance and spectral type assuming an OPD control accuracy comparable to that achieved with the VLTI PRIMA fringe sensing unit. A nulling rejection factor greater than 1000 is obtained for dwarf M, K or G stars at distance greater than 5 pcs. However, due to the high level of the thermal infrared background, the integration time needed to measure the nulling rejection ratio at a $3 \sigma$ accuracy level rapidly increases with distance. Fig.7 (right) shows that hours of integration will be needed to measure the stellar leakage at a rejection level of $1600-1800$ on G-K dwarfs 

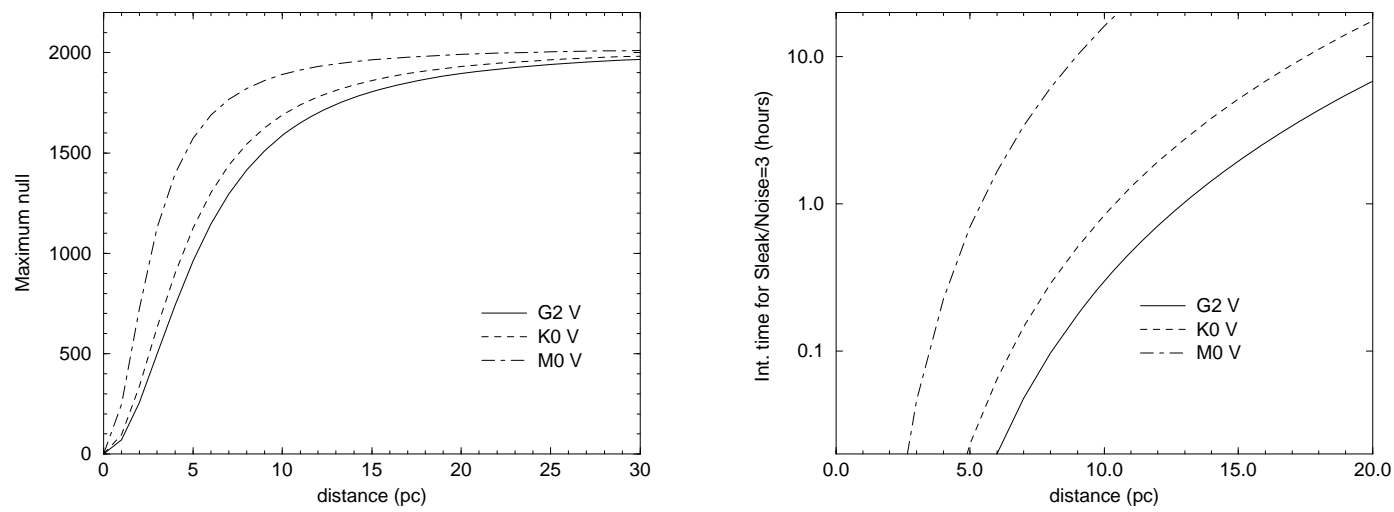

Figure 7. Maximum achievable null (left) in the $\mathrm{N}$ band as a function of star distances and spectral types assuming an OPD control accuracy similar to the ESO VLTI PRIMA fringe sensor unit. Nulling performance better than 1000 could be achieved. However, due to the strong thermal infrared background, the integration time (right) needed for a null depth measurement at a $3 \sigma$ level accuracy in the $\mathrm{N}$ band increases rapidly with the target distance.

at 20 pcs. This estimate does not take into account additional performance limitation by the sky background fluctuations. In order to measure a null depth of a few thousands, the background flux has to be subtracted with an extremely high accuracy in the $\mathrm{N}$ band. In the L' band, the requirement on the background calibration accuracy is relaxed since the background flux is much smaller. The background thermal emission contributes to the noise not only by its photon noise but also by its temporal fluctuations. Eq. 7 assumes that chopping is efficient enough to remove background fluctuations. In practice, little is known about the stability of the background in the mid-IR both on long and short time scales. A measurement campaign of thermal infrared emission and atmospheric dispersion has been conducted using the MID-Infrared instrument (MIDI; Leinert et al. 2003) at the ESO VLTI. Analysis results (Bakker et al. 2004) show important background fluctuations in the $\mathrm{N}$ band on very short timescales, thus ruling out the possibility to calibrate the thermal background emission with a high accuracy in the $\mathrm{N}$ band.

\subsection{The atmospheric dispersion}

A wideband interferometer such as GENIE is sensitive to the effect of longitudinal dispersion which affect the interfering light beams unequally. For infrared observations, this effect is due to the unbalanced air paths in the delay line and to random atmospheric humidity variations in the line-of-sight to the star and inside the VLTI delay line tunnels. Longitudinal dispersion determines the position of the null fringe as a function of wavelength, and is therefore a central problem in producing deep broadband interferometric nulls. It produces two deleterious effects, namely (i) an inter-band dispersion between the spectral band where the OPD is controled and the measurement band where the null is achieved and (ii) an intra-band dispersion within the measurement band. Also, random fluctuations of water vapour content in the path of the beams will lead to a random motion of the dark fringe. This needs to be compensated e.g. by periodically measuring the fringe position in the L' band. Dry air has very little dispersion in the infrared. Water vapour, on the contrary, has a strong dispersion in the infrared, and the column densities of water vapour above the telescopes can differ enough to contribute significantly (Meisner \& Le Poole 2003). Dispersion correction can be achieved with ZnSe plates combined with delay lines (Koresko et al. 2003) providing that a high degree of symmetry is kept in the nuller design to minimize unbalanced reflectivity, transmission or polarisation in the optical system. A pair of wedges is the prefered solution to adjust pathlength through ZnSe while keeping constant the direction of the beams. 

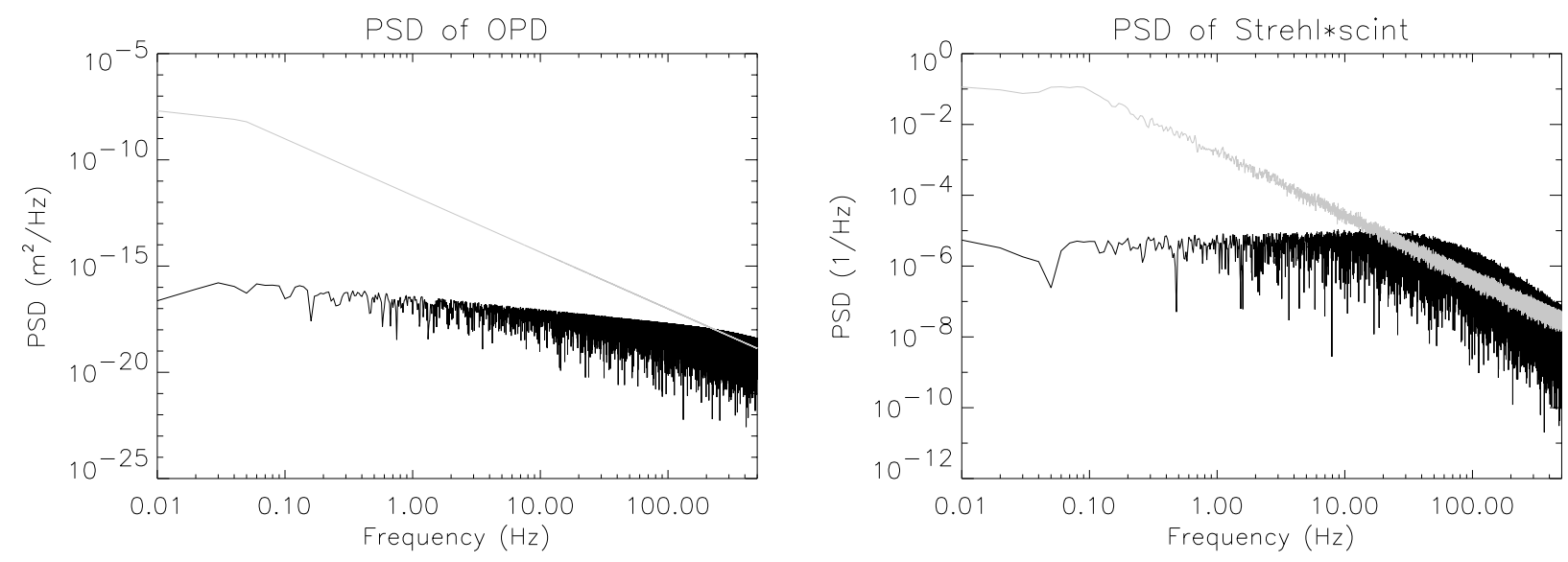

Figure 8. Simulation examples of the power spectral densities of the optical path difference (top) and Strehl fluctuations (bottom) before and after correction by OPD and amplitude control loops of the GENIE experiment.

\section{GENIE NULLING PERFORMANCE}

A simulation software has been developed (Absil et al. 2003a) in an IDL based code to simulate nulling tests and observation scenarios that could be conducted with the GENIE experiment. The code emulates the brightness distribution of the target source including the central star, its circumstellar dust disk and the eventual presence of a low-mass companion. The noise contribution from the thermal infrared emission of the atmospheric background and of the VLTI is added to the signal. A Kolmogorov power spectrum describes the effect of the atmospheric turbulence on the fluctuation of the optical path difference between the telescopes. The GENIE instrument is described by the transfer functions of its different subsystems. This includes the control loops of the MACAO adaptive optics and of the PRIMA fringe sensor unit of the ESO Very Large Telescope Interferometer. Examples of the power spectrum of the OPD and Strehl fluctuations are given in Fig. 8 before and after filtering by the OPD control loop and amplitude matching subsystems of the experiment. The output signal of the GENIE simulator consists in time series of fluxes calculated in different spectral channels by folding the brightness distribution of the source with instantaneous transmission map of the GENIE experiment distorted by OPD, amplitude mismatch and background offsets. Examples of simulation results are given in Fig.9 (left) which shows the cumulative averaged nulled signal after background subtraction in three different channels as a function of integration time. In this example a moderately stable null of about 1500 is achieved in a thousand second.

Table 1. Effect of RMS OPD fluctuations, amplitude fluctuation and thermal infrared background noise on the nulling depth and signal to noise ratio of the stellar leakage measurement. A 100 seconds nulling test is assumed on a A0 V star located at 50 parsecs. Only one spectral channel of $1 \mu \mathrm{m}$ width centered at $3.8 \mu \mathrm{m}$ in the L' band is considered assuming no performance degradation by dispersion effect.

\begin{tabular}{|ccc|cc|}
\hline $\begin{array}{c}\sigma_{\text {opd }} \\
(\mathrm{nm})\end{array}$ & $\begin{array}{c}\sigma_{a m p} \\
(\%)\end{array}$ & $\begin{array}{c}\text { bckgd } \\
\left(\mathrm{e}-\mathrm{s}^{-1}\right)\end{array}$ & Null & $\mathrm{S} / \mathrm{N}$ \\
\hline 0 & 0 & 0 & 2290 & 1570 \\
0 & 0 & $9.7 \times 10^{6}$ & 2290 & 79 \\
0 & 2.5 & 0 & 1690 & 60 \\
20 & 0 & 0 & 1980 & 77 \\
20 & 2.5 & 0 & 1510 & 56 \\
20 & 2.5 & $9.7 \times 10^{6}$ & 1510 & 50 \\
\hline
\end{tabular}



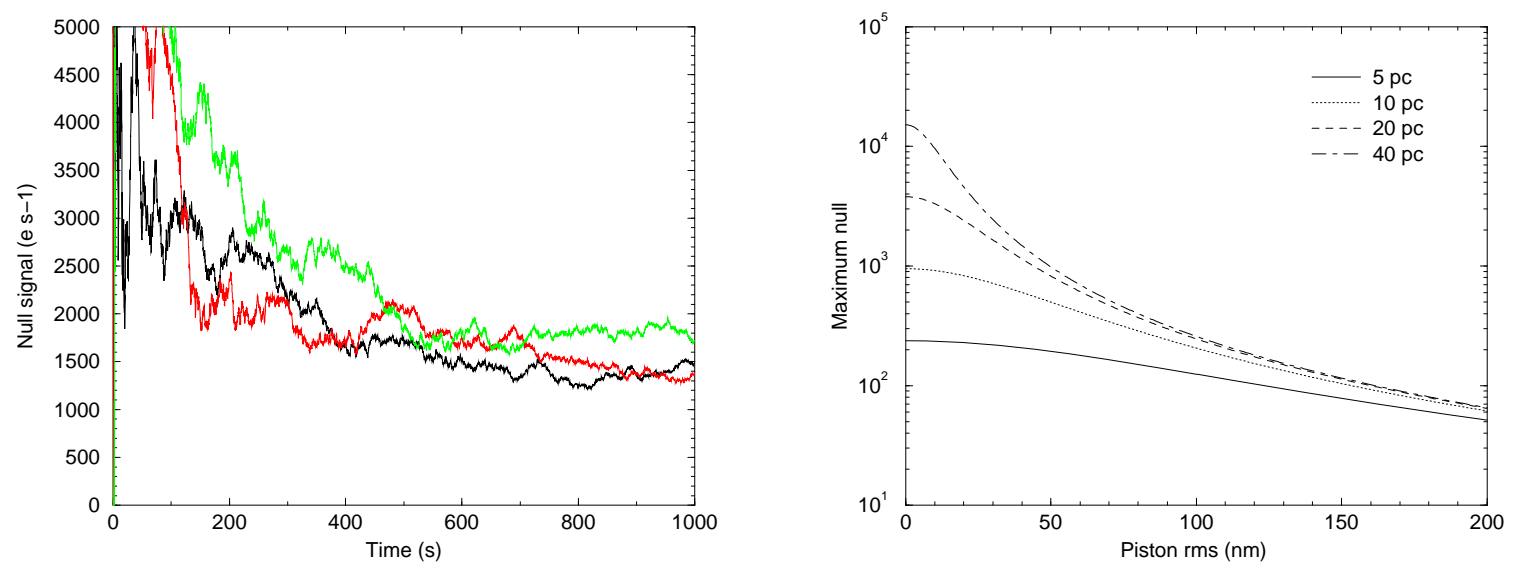

Figure 9. Left: Geniesim simulation example of a cumulative averaged null signal in three different spectral channels.Right: maximum achievable rejection ratio in the L' band as a function of the residual rms piston error for a G2 V star at 5,10, 20 and 40 pc. A two UTs telescope interferometer is assumed with a 46 meter baseline.

One major performance limitation of a Bracewell interferometer operating on nearby stars is due to starlight leakage through the transmission map. The results of a parametric performance analysis model are summarized in Fig.9 (right) which gives the maximum achievable rejection ratio in the L' band as a function of the residual rms piston error for a G2 V star at 5, 10, 20 and 40 pc. A baseline of 46 meter is assumed between two UT telescopes. The nulling performance are severely limited by stellar leakage for stars closer than 10 parsecs. For more distant stars, the maximum achievable null is rapidly dropping with the degradation of the OPD control accuracy. The OPD control loop is a major subsystem of the GENIE experiment and drives the nulling performance of the interferometer in the $\mathrm{L}$ band. This control loop includes a fringe sensor unit and a delay line. The presence of a bright star in the center of the field of view of the nulling interferometer enable to control the optical path difference between the two arm of the interferometer with an accuracy high enough to achieve a nulling depth greater than a thousand.

In order to avoid a degradation of the nulling performance by the wavefront errors, GENIE will use monomode optical fibers or waveguides. These devices will filter the high order wavefront errors that will remain after correction by the VLTI adaptive optics subsystem. Hence, the coherent flux used for destructive interference can be assumed to be the fraction of the total flux equal to the Strehl ratio. The Strehl ratio is expected to fluctuate rapidly due to atmospheric turbulence, therefore affecting the nulling performance. The amplitude fluctuation will have to be corrected by an amplitude matching device. Table 1 quantifies the effect of rms OPD variations, rms amplitude fluctuations and thermal infrared background noise on the nulling depth and signal to noise ratio of the stellar leakage measurement. A 100 seconds nulling test on a A0 V star located at 50 parsecs is considered. A spectral channel width of $1 \mu \mathrm{m}$ has been used in the calculation assuming no performance degradation by dispersion effects. Fluctuation of the thermal infrared background emission are not taken into account. Table 1 indicates that an amplitude control with a percent accuracy is sufficient to maintain a nulling rejection ratio larger than 1000 . Such a performance is feasible in the L' band.

\section{DARWIN-GENIE: A GENERAL USER INSTRUMENT}

The nulling interferometry technique in the infrared is well suited to study any faint cool object located in the immediate environment of a bright astrophysical source (e.g. Voit 1997). A wide range of studies could potentially be conducted using the Darwin-GENIE experiment including infrared spectroscopy of cool dwarfs in spectroscopic binaries, of protoplanetary disks around T Tauri stars, of Herbig Haro objects (Malbet \& Bertout 1995) and of Young Stellar Object in general (Kaltenegger et al. 2003). Such programs would be important 
scientific by-products of the Darwin-GENIE experiment. As an example, a large number of main sequence stars are known to harbor a large quantity of dust. They are mainly early-type stars called Vega type systems after the first discovery of an excess infrared flux around Vega by the satellite IRAS (Aumann et al. 1984). Absil et al. (2003b) simulated observations of a typical Vega-type star ( $\zeta$ Lep) using the GENIE simulation software. The results show that GENIE observations could provide an unprecedented accuracy in the description of the disk morphology including constraints on the dust density and radial temperature distribution. The L' band can be used for such studies with the VLTI UT telescopes. The auxilary telescopes that are dedicated to interferometry would also provide valuable information in the L' band. The simulations also show that GENIE could detect disks as faint as 25 times the solar zodiacal cloud brightness around nearby solar type stars. This would allow to prepare the Darwin science program by discarding unsuitable targets for Earth like planet detection by IR nulling interferometry from space (see Sect. 2.2).

Since 1995, more than 100 extrasolar planets have been discovered as companions to solar-type stars (Marcy et al. 2000). All of these planets were discovered indirectly in high-precision Doppler surveys. These surveys

measure the radial velocity Doppler shift of the parent star due its periodic motion around the center of mass of the star plus planet system. This technique is most sensitive to massive planets with short orbital periods. The extrasolar planets that have been discovered today have mass ranges from $M \times \operatorname{sini} \approx 0.15$ to 10 Jupiter masses (where $i$ is the orbit inclination onto the observer line of sight) with orbital periods ranging from 3 days to a few years. Because of their proximity to the parent star $(<<1 \mathrm{AU})$, the atmosphere of some of these planets is much hotter than that of Jupiter (and of the Earth), hence their name, hot Jupiters. With temperatures sometimes higher than $1000 \mathrm{~K}$, their thermal emission is maximum in the near infrared bands. With baselines ranging from 46 to $130 \mathrm{~m}$, the VLTI is optimized for the detection in the L' band of planets at about 5-15 milli-arcsec from their parent star which correspond to 0.05-0.15 AU for a nearby star at 10 parsecs. This is specially fortunate since these planets are precisely the hot Jupiters of which a significant sample is known. The contrast between a hot Jupiter and its parent star is reduced to less than $10^{4}$ in the L'band. Den Hartog et al. (2003) simulated an observation of the $\tau$ Boo system using the GENIE experiment. They found that for the most suitable baseline, the starlight leakage is higher than the hot-Jupiter signal. They concluded that one major difficulty in the detection of hot Jupiter from ground will be to calibrate the stellar leakage with a high accuracy.

\section{CONCLUSION}

The Darwin-GENIE nulling experiment will provide European Scientists and Engineers with a first experience on the design , manufacture and operation of a nulling interferometer using Darwin representative concept and technology. Nulling tests on single stars or close binary systems can be achieved in the L' band with a nulling rejection ratio higher than a 1000. The Darwin-GENIE experiment will combine all optical functions foreseen into the future Darwin Infrared Space Interferometer. It will demonstrate that nulling interferometry can be achieved in a broad mid-IR band as a precursor to the next phase of the Darwin program.

GENIE is not only a technology demonstrator but also a preparatory experiment for the Darwin science program. The instrument will detect bright exo-zodiacal dust disks around nearby solar type stars. This would allow to establish the Darwin target list and to discard unsuitable sources for Earth like planet detection from space. Darwin observation scenarios, calibration procedures and data analysis methods will be tested by GENIE. The nulling experiment could also become a very useful instrument for astrophysics that would enable scientist to conduct research programmes on a variety of sources including Herbig Haro and Young Stellar Objects, protoplanetary disks around T Tauri stars, low mass companions and maybe hot Jupiters around nearby stars.

\section{REFERENCES}

1. Absil O, Gondoin P., den Hartog R., Erd C., Fridlund M., Rando N., 2002, in the Proceedings of La Semaine de l'Astrophysique Francaise, meeting held in Paris, France, June 24-29, 2002, Eds.: F. Combes and D. Barret, EdP-Sciences (Editions de Physique), Conference Series, p. 145

2. Absil O, den Hartog R., Erd C., Gondoin P., Kaltenegger L., Fridlund M., Rando N., Wilhelm R., 2003a, In: Proceedings of the Conference on Towards Other Earths: DARWIN/TPF and the Search for Extrasolar Terrestrial Planets, 22-25 April 2003, Heidelberg, Germany. ESA SP-539, 2003, p.317. 
3. Absil O, Kaltenegger L., Eiroa C., den Hartog R., Gondoin P., Fridlund M., Wilhelm R., 2003b, In: Proceedings of the Conference on Towards Other Earths: DARWIN/TPF and the Search for Extrasolar Terrestrial Planets, 22-25 April 2003, Heidelberg, Germany. ESA SP-539, 2003, p.323.

4. Angel J.R., Cheng A.Y., Woolf N.J., 1986, Nature 322, 341

5. Backman D.E., Parece F., 1993, in Protostar and Planet III, ed. E.H. Levy \& J.I. Lunine (Tucson, Univ. of Arizona Press), 1253

6. Bakker E. et al. 2004, in these proceedings

7. Barillot M., 2004, In: Proceedings of the Conference on Towards Other Earths: DARWIN/TPF and the Search for Extrasolar Terrestrial Planets, 22-25 April 2003, Heidelberg, Germany. ESA SP-539, 2003, p.345.

8. Bracewell R.N., 1978, Nature 274, 780

9. den Hartog R., Absil O., Kaltenegger L., Gondoin P., Wilhelm R., Fridlund M., 2003, In: Proceedings of the Conference on Towards Other Earths: DARWIN/TPF and the Search for Extrasolar Terrestrial Planets, 22-25 April 2003, Heidelberg, Germany. ESA SP-539, 2003, p.399.

10. Eiroa C. et al., 2003, In: Proceedings of the Conference on Towards Other Earths: DARWIN/TPF and the Search for Extrasolar Terrestrial Planets, 22-25 April 2003, Heidelberg, Germany. ESA SP-539, 2003, p.403.

11. ESA, 2000. Darwin: the Infrared Space Interferometer: Concept and Feasibility Study Report ESASCI $/ 2000 / 12$

12. Flatscher R., 2003, In: Proceedings of the Conference on Towards Other Earths: DARWIN/TPF and the Search for Extrasolar Terrestrial Planets, 22-25 April 2003, Heidelberg, Germany. ESA SP-539, 2003, p.283.

13. Fridlund M. 2000, in Proceedings of the Conference 'Darwin and Astronomy - the Infrared Space Interferometer', Stockholm, Sweden, 17-19 November 1999, ESA SP-451

14. Fridlund M., Gondoin P. 2003, SPIE Vol. 4852, p. 394

15. Gondoin P., Absil O., Fridlund M., Erd C., den Hartog R., Rando N., Glindemann A., Koehler B., Wilhelm R., Karlsson A., Labadie L., Mann I., Peacock A., Richichi A., Sodnik Z., Tarenghi M., Volonte S., 2003, SPIE Vol. 4838, p. 700

16. Kaltenegger L., Absil O., Eiroa C., et al. 2003, In: Proceedings of the Conference on Towards Other Earths: DARWIN/TPF and the Search for Extrasolar Terrestrial Planets, 22-25 April 2003, Heidelberg, Germany. ESA SP-539, 2003, p.465.

17. Koresko C. D., Mennesson B. P., Serabyn E., Colavita M., Akeson R. L., Swain M. R., 2003, Proc. SPIE 4838, pp. 625-635

18. Küchner M. J., Brown M.E., 2000, PASP 112, 827

19. Leinert C., Graser U., Waters L. B. F. M, Perrin G. S. et al., 2003, Proc. SPIE 4838, pp. 893-904

20. Malbet F., Bertout C., 1995, A\&AS, 113, 369

21. Marcy G.W., Cochran W.D., Mayor M., 2000, in Protostar and Planet IV, ed. V. Mannings, A.P. Boss \& S.S. Russel (Tucson, Univ. of Arizona Press), 1285

22. Meisner J., Le Poole R., 2003, Proc. SPIE 4838, pp. 609-623

23. Ollivier M., Mariotti J.M., 1997, App. Opt. 365340

24. Ruilier C., 1998, Proc. SPIE 3350, p. 319

25. Serabyn E. 2003, In: Proceedings of the Conference on Towards Other Earths: DARWIN/TPF and the Search for Extrasolar Terrestrial Planets, 22-25 April 2003, Heidelberg, Germany. ESA SP-539, 2003, p.91.

26. Shaklan S., Roddier F., 1988, App. Opt. 27, 2334

27. Schöller M., Glindemann A., 2003, In: Proceedings of the Conference on Towards Other Earths: DARWIN/TPF and the Search for Extrasolar Terrestrial Planets, 22-25 April 2003, Heidelberg, Germany. ESA SP-539, 2003, p.109.

28. Voit M. G., 1997, ApJ 487, L109 